\title{
Can the 72-hour rule based on "Blast/Abn Lymph" flag on Sysmex XN-10 optimize the workflow in hematology laboratory?
}

\author{
La règle 72 heures basée sur l'alarme "Blast/Abn Lymph" \\ du Sysmex XN-10 permet-elle d'optimiser le flux de travail \\ dans les laboratoires d'hématologie?
}

\author{
Henry Paridaens ${ }^{1, a}$ \\ Lina Sabor ${ }^{1, a}$ \\ Joanna Simar ${ }^{1, a}$ \\ Emily Ronez ${ }^{2}$ \\ Edouard Cornet ${ }^{3}$ \\ Jean-Philippe Defour ${ }^{1}$ \\ ${ }^{1}$ Department of laboratory \\ medicine-hematology, Cliniques \\ universitaires Saint-Luc and Université \\ catholique de Louvain, Brussels, \\ Belgium \\ 2 Department of laboratory \\ medicine-hematology, Assistance \\ publique-Hôpitaux de Paris, Paris, \\ France \\ ${ }^{3}$ Laboratory of hematology, \\ Centre hospitalier universitaire, \\ Caen, France \\ a The authors contributed equally \\ to this work.
}

\begin{abstract}
Despite the continuing improvement of automated blood cell counters, confirmation by blood smear examination remains the gold standard in case of anomalies. With a constant goal of standardisation, different experts committees (e.g. the French-speaking cellular hematology group (Groupe francophone d'hématologie cellulaire, GFHC and the ISLH International society for laboratory hematology) recently published criteria for microscopic analysis of blood smears. Cornet et al. evaluated the application of those criteria and propose to suppress any review for 72 hours when a "Blast/Abn lymph" flag is triggered for a sample with no abnormal cell on the microscopic review. The aims of our study were to retrospectively evaluate whether this 72-hour rule adequately operates and whether it is possible to extend the arbitrary 72-hour timeframe to $96 \mathrm{~h}$ and $144 \mathrm{~h}$. To achieve this goal, 40,688 blood samples were collected from three French-speaking hospitals. 1,548 samples presented an isolated "Blast/Abn lymph" flag. Only 221 samples presented the application of the 72-hour rule at least once for our study period. We were able to extend this rule to 144 hours for 10 samples of them. All blood smears for which the rule was applied were verified and there was no abnormal cell on smears at 72 and 144 hours. In conclusion, the 72-hour rule derived from the GFHC's criteria is secure and reduces the slide review rate and thus the production costs and the turnaround time of hemogram results. Further investigations could confirm that its extension to 144 hours is also adequate.
\end{abstract}

Key words: flag, 72-hour rule, Blast/Abn lymph, blood smear, XN-10

Résumé. Malgré l'amélioration continue des automates d'hématologie cellulaire, la confirmation des cas pathologiques par microscopie demeure la technique de référence. Dans un objectif constant de standardisation, différents comités d'experts (dont le GFHC, Groupe francophone d'hématologie cellulaire et I' ISLH, International society for laboratory hematology) ont récemment publié des critères de revue microscopique des frottis sanguins. Cornet et al. ont évalué l'application de ceux-ci et proposent de supprimer toute revue microscopique durant une période de 72 heures lorsqu'une alarme isolée «Blast/Abn Lymph » est déclenchée pour un échantillon dont la revue microscopique ne révèle aucune cellule anormale. Les buts de notre étude sont d'évaluer rétrospectivement l'application correcte de la règle 72 heures ainsi que sa possible extension à 96 et 144h. Pour ce faire, 40688 échantillons sanguins ont été collectés dans 3 hôpitaux francophones ; 1548 échantillons présentaient une alarme isolée "Blast/Abn lymph" et seuls 221 échantillons ont présenté une application de la règle 72 heures au minimum une fois durant la période de notre 
étude. Par la suite, nous avons pu étendre la règle à $144 \mathrm{~h}$ pour 10 échantillons. Tous les frottis sanguins pour lesquels la règle avait été appliquée ont été revus au microscope et aucune cellule anormale n'a été observée à 72 et 144 heures. En conclusion, la règle 72 heures dérivant des critères du GFHC est sûre. Elle réduit le taux de revue microscopique, donc les coûts engendrés et le délai de transmission des résultats. Des investigations complémentaires sont nécessaires afin de confirmer la fiabilité de l'extension à 144h.

Mots clés : alarme, règle 72 heures, Blast/Abn lymph, frottis sanguin, $\mathrm{XN}-10$

Although advances have been continuously made to automated blood cell counter technology, confirmation by blood smear examination under microscopy remains the gold standard when automated instruments detect anomalies. Flags triggering microscopic blood smears vary widely between manufacturers, however, the "blast" flag is the most commonly used. It is generally very sensitive, despite a lack of specificity and a high rate of false positives. Clinical laboratories need to standardise their procedures by making their daily practices consistent, including the guidelines leading to a smear.

To achieve this goal, different expert committees (e.g. the French-speaking cellular hematology group (Groupe francophone d'hématologie cellulaire, GFHC and the ISLH International society for laboratory hematology) established quantitative and/or qualitative criteria. These minimal recommendations are intended to keep the number of blood smears at a manageable level, provide added clinical value for the prescribing physician and retain elevated diagnostic sensitivity.

Cornet et al. evaluated GFHC's criteria on the XN-10 analyser system (Sysmex Corporation, Kobe®, Japan) [1]. They proposed four modifications to optimise patient care with increased efficiency and reduced costs. One of the modifications involves suppressing any microscopic white blood cells (WBC) slide review for 72 hours in the context of a recurrent and isolated false positive "Blast/Abn lymph" flag [2].

This new criterion implies that no other rule appears in the course of a new blood differential.

The aims of our study were to evaluate whether the "Blast/Abn lymph" rule adequately operates within 72 hours and whether it is possible to extend this arbitrary timeframe.

\section{Materials and methods}

\section{Study design}

This study was performed in three French-speaking hospitals: Cliniques universitaires Saint-Luc (Brussels, Belgium), CHU Ambroise Paré (Boulogne-Billancourt,
France) and CHU Caen (Caen, France) for a one-month period.

Cliniques universitaires Saint-Luc is a university hospital. It is one of the largest hospitals in Brussels with 979 beds and more than 500,000 outpatient visits per year. It has a large oncology and haematology department. Its haematological laboratory analyses around 250,000 complete blood counts annually.

CHU Ambroise Paré has a total capacity of 595 beds. It also performs blood differentials for Institut Curie (site René Huguenin), a specialised site with 146 beds and a dedicated onco-haematology ward. Its haematological laboratory annually performs 85,000 blood differentials.

CHU Caen is a 1,582 bed hospital representing all clinical specialties including neonatology and oncology, as well as a specialised haematology ward. The laboratory performs around 240,000 cells blood analyses a year.

In a total cohort of 40,688 blood samples, we studied over a 6-day period (144 hours) patients for whom the 72-hour timeframe protocol was effective. Those samples were obtained from our three hospitals' daily routine over a one-month period. The study included both inpatients and outpatients. Approximately $13 \%$ of all samples came from a haematology or oncology department.

Blood samples were collected in EDTA K3 Sarstedt Monovette $^{\mathrm{TM}}$ (Sarstedt, Nümbrecht, Germany) at Cliniques universitaires Saint-Luc and EDTA K2 from Becton Dickinson $^{\mathrm{TM}}$ (Franklin Lakes, New Jersey, United States,) in CHU Ambroise Paré and Caen.

The pre-analytical phase protocols were compliant with standard recommendations and blood samples were analysed within six hours of collection. All the blood differentials were performed on XN10 analysers controlled by Extended IPUs (Information processing unit Version 3.1.7.0) modules, integrating the manufacturer's recommendations and GFHC criteria for validation rules. All systems were calibrated by Sysmex and controlled during the study by internal quality controls.

$\mathrm{XN}-10$ analysers (Sysmex corporation ${ }^{\mathrm{TM}}$, 1-5-1, Wakinohamakaigandori, Chuo-ku, Kobe, Hyogo 651-0073, Japan) establish a white blood cells differential via cytometry and fluorescence combining two channels; WBC differential (WDF) and WBC count (WNR) channels. 


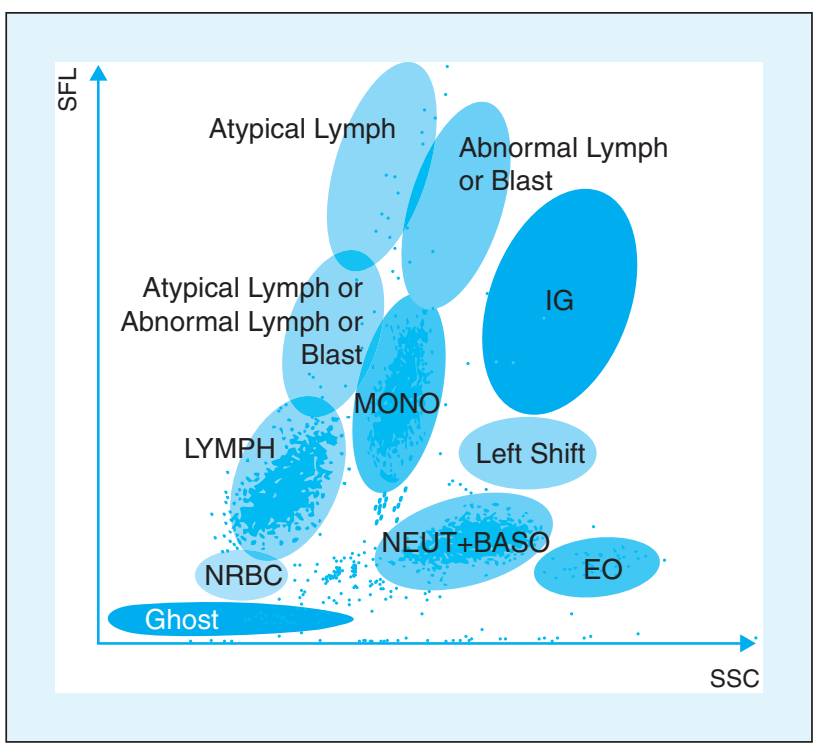

Figure 1. Scattergram of WDF channel of XN 10 with flagging areas.

XN-10's WDF channel measures mature neutrophils, lymphocytes, monocytes, eosinophils and immature granulocytes (promyelocytes, myelocytes and metamyelocytes). The WNR channel provides the basophils and NRBC (nucleated red blood cells count). These analysers also provide qualitative flags derived mainly from the WDF channel, in particular the "Atypical Lymph" flag (suspicion of reactive lymphocytes), the "Blast/Abn lymph" flag (suspicion of blast cells or abnormal lymphocytes) and the abnormal leukocyte scattergram flag (which highlights the inability to separate different cell populations). All these flags lead to a smear revision according to the manufacturer's recommendations (figure 1).

\section{Criteria set for blood smear review}

When considering the criteria set for blood smear reviews, we distinguish two major types of rules. On the one hand, there are manufacturers' rules that are not adaptable by laboratory staff. These procedures ensure mainly technical validation and relate to flagging parameters, pre-analytical rules and a suspicion of invalid results. Secondly, there is the Extended IPU module that embeds the quantitative and qualitative criteria and conditions issued from the GFHC recommendations [2].

\section{Blood smear review}

All the blood smears for which the 72-hour rule was applied were verified.

Blood smear reviews were performed either by Sysmex ${ }^{\circledR}$ DI-60 system (DI-60, Sysmex, Kobe, Japan) on 200 cells or by manual microscopy depending on the site.
In absence of abnormal cells and/or morphological comment, the WBC count from the WNR channel must be accepted to trigger the 72-hour rule.

In presence of abnormal cells, the WBC count from the manual count or from the reclassification of the DI-60 must be accepted and therefore the 72-hour rule cannot apply.

\section{Definition of sensitivity and specificity of the "Blast/Abn lymph" analyser flag}

Samples were defined as true positive (TP) when the "Blast/Abn lymph" flag was present and the presence of malignant cells was confirmed by a microscopic examination.

Samples were defined as false negative (FN) when the "Blast/Abn Lymph" flag was absent despite the presence of malignant cells on smear examination.

Samples were defined as true negative (TN) when the "Blast/Abn lymph" was absent and no malignant disease was known in the medical record or no malignant cells were observed on microscopic examination.

Samples were defined as false positive (FP) when the "Blast/Abn lymph" flag was present in absence of malignant cells.

Sensitivity (Se) and specificity (Sp) were calculated according to the following equations:

$$
\begin{aligned}
& \mathrm{Se}=\mathrm{TP} /(\mathrm{TP}+\mathrm{FN}) \\
& \mathrm{Sp}=\mathrm{TN} /(\mathrm{TN}+\mathrm{FP})
\end{aligned}
$$

Sensitivity can be defined as the accuracy of the "Blast/Abn Lymph" flag to identify the malignant cells whereas specificity shows the trustworthy absence of this flag for samples without malignant cells.

\section{2-hour rule definition and analysis}

The 72-hour rule allows the delay of slide review for 72 hours if there is a recurrent and isolated "Blast/Abn lymph" flag and if no abnormal cell has been seen on the first slide. If any other flag appears during the 72-hour rule, this rule is automatically cancelled and a slide review is required. The analysis preceding the first cancelled smear is considered time 0 . The 72 -hour rule can only apply if the WBC count from the WNR channel is accepted after the slide review.

\section{Results}

\section{Phase 1: Evaluation of the "Blast/Abn lymph" analyser flag}

For the 40,688 samples studied in February 2017, 4,275 WBC smears (10.5\% of total samples) were generated by WBC rules in which 2,846 (66.6\% of WBC smears) presented a "Blast/Abn lymph" analyser flag. For 1,548 


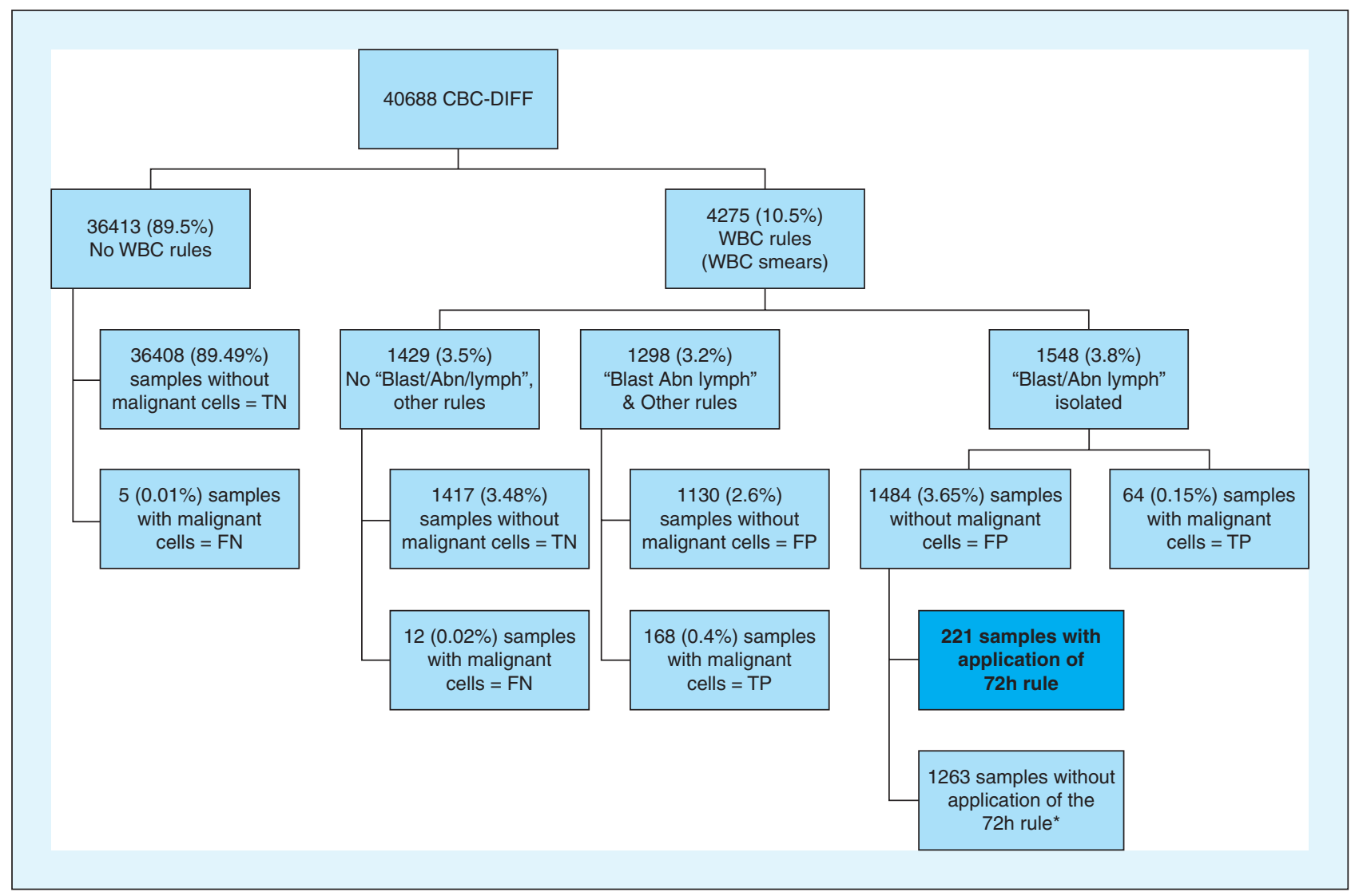

Figure 2. Distribution of the analyzer flags amongst our 40,688 complete blood. TN: true negative, FN: false negative, FP: false positive, TP: true positive. * The absence of application of the $72 \mathrm{~h}$ rule for those samples could be explained by the absence of follow-up samples or by the presence of other WBC flags (e.g. neutropenia ....) for the subsequent samples cancelling the application of the $72 \mathrm{~h}$ rule.

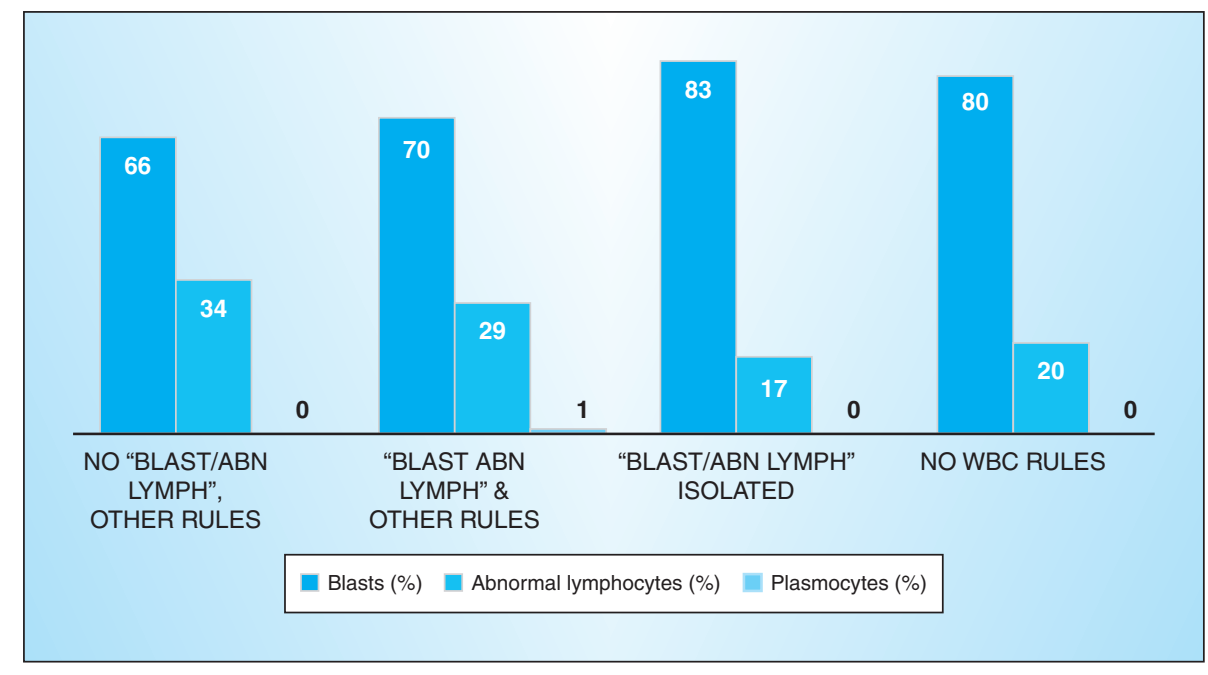

Figure 3. Distribution of pathological cells for all the samples with malignant cells.

samples $(36.2 \%$ of WBC smears, $3.8 \%$ of total samples) this "Blast/Abn lymph" flag was isolated and not associated with others analytical criteria (figure 2).
During our study, $5.7 \%$ of smears triggered by WBC rules $(244 / 4,275)$ presented malignant cells. Amongst those slides, 168 (68.9\% of malignant samples) were triggered by 
"Blast/Abn lymph" and other rules, 64 (26.2\% of malignant samples) by an isolated "Blast/Abn lymph" and 12 (4.9\% of malignant samples) by another rule than "Blast/Abn lymph".

5 samples of the 36,413 with no WBC rules presented malignant cells which were found after medical request ( $2 \%$ of malignant samples; $0.01 \%$ of total samples). A thorough examination of those five undetected malignant samples revealed that 4 of them were positive for blasts and 1 of them was positive for abnormal lymphocyte. Those samples resulted from follow-up with a previous positive result for pathological cells. In all the other samples with a "Blast/Abn lymph" flag, 8.1\% actually revealed malignant cells (232/2846). (figures $2 \& 3$ ).

Finally, we evaluated the performances of the "Blast/Abn lymph" flag according to the previous definition of true positive, false negative, true negative and false positive samples of this analyser flag. The flag demonstrated good sensitivity (93\%) and specificity (94\%) (figure 2).

\section{Phase 2: Evaluation the 72-hour rule}

Due to the absence of follow-up for all samples, in particular for neonates, only 221 samples, corresponding to 154 patients, were studied during 72 hours. In those 221 samples, the 72-hour rule applied at least once. It is noteworthy that up to $11.3 \%(25 / 221)$ of those studied samples have benefited from the full-time frame of the rule and were all negative at 72 hours (table 1 ).

In total, the application of this cancelation rule allows us to avoid $14.3 \%(221 / 1,548)$ of smears with isolated "Blast/Abn lymph" in a 72-hour period, which represents $5.1 \%$ of smears for positive WBC criteria.

\section{Phase 3: Optimization of the 72-hour rule}

As we extend the 72 -hour rule to 144 hours $(144 \mathrm{~h}$ from $\mathrm{t}=0 \mathrm{~h}$; smear cancel sequence), 10 samples still present the 144hour rule $(0.6 \%$ of samples with isolated flag "Blast/Abn lymph") over the complete time frame and were negative at 144 hours (table 1).

We can avoid up to $15.9 \%$ of smears with isolated "Blast/Abn lymph" if the 144-hour timeframe is applied without any false negative samples; this represents $5.7 \%$ $(246 / 4,275)$ of smears for positive WBC criteria (table 1).

\section{Phase 4: Predominant population for which the 72-hour "Blast/Abn lymph" rule applies}

The frequency of the "Blast/Abn lymph" flag was higher in neonates and young children ( $<5$ years old) and begins to increase weakly after 40 years with a minimal occurrence in the 20-40 years age bracket (table 2).

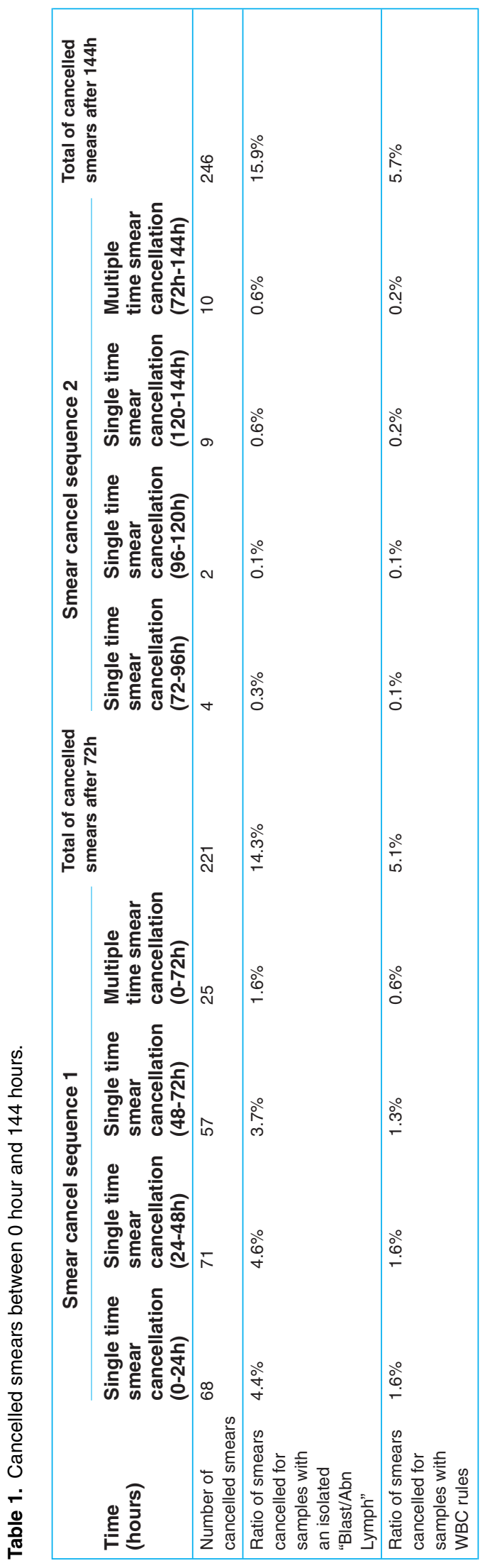


Table 2. Ratio of samples with "Blast/Abn lymph" flag per age category.

\begin{tabular}{|c|c|c|c|}
\hline & $\begin{array}{l}\text { Total } \\
\text { samples }\end{array}$ & $\begin{array}{l}\text { Samples with } \\
\text { Blast/Abn Lymph flag } \\
\text { in the group }\end{array}$ & $\begin{array}{l}\text { Samples with isolated } \\
\text { Blast/Abn Lymph flag } \\
\text { in the group }\end{array}$ \\
\hline$<2$ years & $3.0 \%$ & $50.3 \%$ & $28.7 \%$ \\
\hline 2 years $\leq$ age $<5$ years & $1.5 \%$ & $33.7 \%$ & $20.9 \%$ \\
\hline 5 years $\leq$ age $<10$ years & $1.5 \%$ & $14.2 \%$ & $9.3 \%$ \\
\hline 10 years $\leq$ age $<20$ years & $3.7 \%$ & $8.1 \%$ & $4.2 \%$ \\
\hline 20 years $\leq$ age $<30$ years & $6.3 \%$ & $3.4 \%$ & $1.8 \%$ \\
\hline 30 years $\leq$ age $<40$ years & $8.0 \%$ & $2.9 \%$ & $1.9 \%$ \\
\hline 40 years $\leq$ age $<50$ years & $9.4 \%$ & $4.3 \%$ & $2.2 \%$ \\
\hline 50 years $\leq$ age $<60$ years & $13.6 \%$ & $5.5 \%$ & $2.6 \%$ \\
\hline 60 years $\leq$ age $<70$ years & $17.9 \%$ & $6.6 \%$ & $3.4 \%$ \\
\hline 70 years $\leq$ age $<80$ years & $15.6 \%$ & $5.1 \%$ & $2.6 \%$ \\
\hline 80 years $\leq$ age & $19.5 \%$ & $4.7 \%$ & $2.5 \%$ \\
\hline
\end{tabular}

Furthermore, this flag is more frequent in neutropenic adult patients. Among the $2.9 \%$ of adults ( $>15$ years) with less than $1.510^{9} / \mathrm{L}$ neutrophils $(1,058 / 37,665)$, the isolated "Blast/Abn lymph" flag was frequent at $12.8 \%$ (127/989) (data not shown). This observation suggests that the "Blast/Abn lymph" flag frequency increases when the neutrophil count decreases, such as for patients treated with chemotherapy.

\section{Discussion}

Although blood smear review is a time-consuming task, it remains essential in some pathological situations. As laboratories are facing an increasing workload, mainly due to accreditation requirements, solutions have to be found to maintain elevated diagnostic sensitivity of blood smear review that copes with local limitations such as budgetary constraints, personnel shortage, staff training and local resources [3-5]. Moreover, the need for standardisation implies that each laboratory must implement rules that, alongside the technical criteria provided by the manufacturer, best fit international recommendations and their own routine.

In our multi-centric study, we chose to implement the new recommendations suggested by the GFHC and we first evaluated the 72-hour rule suggested by Cornet et al. [2]. During the period of our study, no false negative result occurred within the course of this 72-hour rule timeframe. According to our data, the application of the 72-hour rule is reliable and can be implemented in daily practice. However, human expertise is essential in the review of the smear. Ongoing staff training therefore remains a priority to avoid missing pathological cells on slides at 0 hour and the inappropriate application of the 72-hour rule.
This 72-hour rule only applies with an isolated "Blast/Abn lymph" flag. Consequently, its effectiveness decreases over time, in particular due to additional WBC technical flags or recommendation rules (e.g. left shift, atypical lymphocytes, neutropenia). An extension of the arbitrary 72-hour timeframe to 144 hours seems reasonable since all the 10 samples analyses at 144 hours were negative. Though, further investigations are needed to confirm these previous results. In this study, we identified two groups in which "Blast/Abn lymph" flags increase but also affect the 72-hour rule efficiency. The first group is the neonate population where the flag is recurrent and where follow up is rare. Becker et al. reported a low specificity level below $35 \%$ for the flag "Blast/Abn lymph" for neonates and infants in the eight-day to 2-year age range. This can be explained by the physiological lymphocytosis as well as the immature appearance of the lymphocytes observed in this age group, which seem to strongly impact the generation of the "Blast/Abn lymph" flag [6]. The second group is chemotherapy patients with a neutrophil count under $1.010^{9} / \mathrm{L}$ in the course of aplasia. Neutropenia, as a flag, is a frequent cause of interruption of the 72-hour rule application. In this second group, fragility of haematological cells (probably causing a different interaction with Sysmex ${ }^{\mathrm{TM}}$ reagents) and XN's limitation of its adaptative cluster analysis in cases of leucopenia could be responsible for the concomitant increase in the "Blast/Abn lymph" flag. We would recommend considering those two points to optimise the efficiency of the rule cancelation.

We also aimed to estimate the economic impact of the application of the 72-hour rule. Cornet et al. estimated that the cost of a smear review for non-pathological samples was around EUR 7.30 (unpublished data). Smear cancellation by applying the aforementioned rule related to 221 samples for our 1-month study period, which delivers a cost reduction of EUR 1,613 $(7.30 \times 221)$. In extrapolation to a 
year, EUR 19,359 could be saved by applying this rule. This would increase up to EUR 21,549 by applying the 144-hour timeframe $(246$ samples $\times 7.30 \times 12)$.

\section{Conclusion}

The "Blast/abn lymph" derived rule applies and cancels $5.2 \%$ to $5.7 \%$ of smears for positive WBC criteria depending on the 72 hours or 144 hours intervals used.

We could assume that applying the 72-hour "Blast/Abn lymph" rule is reliable as no false negatives were found during our study period. This rule significantly helps to improve the workflow of the haematology laboratory and reduces the production cost and the turnaround time of blood differentials. So far, extending to 144 hours appears to be also reliable, but further investigations are recommended before implementing this new time frame in daily practice. Finally, the accurate application of this rule requires a careful examination of the smear to avoid an initial false negative. Continuous training of the staff is thus of prime importance.

Acknowledgements. We would like to thank Jean-Pierre Perol (Sysmex ® Europe) for providing data relative to rules for slide review and for his valuable help, assistance and availability during our study.
Conflict of interest: none of the authors has any conflict of interest to disclose.

\section{References}

1. Geneviève F, Mercier-Bataille $\mathrm{D}$, Wagner-Ballon $\mathrm{O}$, Trimoreau F, Schillinger F, Leymarie V, et al., pour le Groupe francophone d'hématologie cellulaire. Revue microscopique du frottis sanguin: propositions du Groupe francophone d'hématologie cellulaire (GFHC). Feuillets de Biologie 2014 ; LVI.(317).

2. Cornet E, Mullier F, Despas N, Jacqmin H, Geara C, Boubaya $\mathrm{M}$, et al. Evaluation and optimization of the extended information process unit (E-IPU) validation module integrating the sysmex flag systems and the recommendations of the French-speaking cellular hematology group (GFHC). Scand J Clin Lab Invest 2016;76(6): 465-71.

3. Bain BJ. Diagnosis from the blood smear. $N$ Engl $\mathrm{J}$ Med $2005 ; 353(5): 498-507$.

4. Briggs C, Longair I, Kumar P, Singh D, Machin SJ. Performance evaluation of the Sysmex haematology XN modular system. J Clin Pathol $2012 ; 65(11): 1024-30$.

5. Gulati G, Song J, Florea AD, Gong J. Purpose and criteria for blood smear scan, blood smear examination, and blood smear review. Ann Lab Med 2013;33(1) : 1-7.

6. Becker PH, Fenneteau O, Da Costa L. Performance evaluation of the Sysmex XN-1000 hematology analyzer in assessment of the white blood cell count differential in pediatric specimens. Int J Lab Hematol $2016 ; 38(1): 54-63$. 